\begin{tabular}{|c|c|c|}
\hline 1 & $\begin{array}{c}\text { International Journal of Research in Engineering and Innovation } \\
\text { (IJREI) } \\
\text { journal home page: http://www.ijrei.com }\end{array}$ & 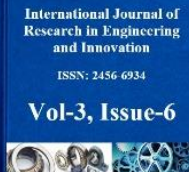 \\
\hline & ISSN (Online): 2456-6934 & Hos. \\
\hline
\end{tabular}

\title{
Evaluation of thermodynamic energy-exergy performances in modified vapour compression refrigeration systems
}

\author{
R.S. Mishra
}

Department of Mechanical, Production, Industrial and Automobiles Engineering, Delhi technological University, Delhi, India

\begin{abstract}
In this investigation the work input required running the vapour compression refrigeration system is reduced by using compound compression and further be decreased by using flash intercooling between compressors. COP of system can also be enhanced by compressing the refrigerant very close to the saturation line this can be achieved by compressing the refrigerants in more stages with intermediate intercoolers. The refrigeration effect can be increase by maintaining the condition of refrigerants in more liquid stage at the entrance of evaporator which can be achieved by expanding the refrigerant very close to the liquid line. The expansion can be brought close to the liquid line by subcooling the refrigerant and removing the flashed vapours by incorporating the flash chamber in the working cycle. The evaporator size can be reduced because unwanted vapours formed are removed before the liquid refrigerant enters in the evaporator. Multi-stage vapour compression with flash intercooler and individual throttle valves (system-1) consists of three compressors arranged in compound compression, individual throttle valves, condenser and evaporators. Multiple evaporators at different temperatures with compound compression, flash intercooler and multiple throttle valves (system-2) consists of three compressors arranged in compound compression, multiple throttle valves, condenser and evaporators. Vapour compression refrigeration system using multiple evaporators at different temperatures with individual compressors, multiple expansion valves (system-3) and vapour compression refrigeration system using multiple evaporators at different temperatures with individual compressors, individual expansion valves (system-4).

(C) 2019 ijrei.com. All rights reserved
\end{abstract}

Keywords: Thermodynamic Analysis, Energy, Exergy Analysis, Irreversibility Evaluation

\section{Introduction}

Refrigeration is a technology which absorbs heat at low temperature and provides temperature below the surrounding by rejecting heat to the surrounding at higher temperature. Simple vapour compression refrigeration system which consists of four major components compressor, expansion valve, condenser and evaporator in which total cooling load is carried at one temperature by single evaporator but in many applications like large hotels, food storage and food processing plants, food items are stored in different compartment and at different temperatures. Therefore there is need of multi evaporator vapour compression refrigeration system. The systems under vapour compression technology consume huge amount of electricity, this problem can be solved by improving performance of system. Performance of systems based on vapour compression refrigeration technology can be improved by following:

The performance of refrigerator is evaluated in term of COP which is the ratio of refrigeration effect to the net work input given to the system. The COP of vapour compression refrigeration system can be improved either by increasing refrigeration effect or by reducing work input given to the system. The throttling process in VCR is internal irreversible expansion process because expansion process is one of the main factors responsible for exergy loss in cycle performance because of entering the portion of the refrigerant flashing to vapour in evaporator which will not only reduce the cooling capacity but also increase the size of evaporator. This problem can be eliminated by adopting multi-stage expansion where the flash vapours is removed after each stage of expansion as a consequence there will be increase in cooling capacity and reduce the size of the evaporator. 


\subsection{Use of ecofriendly HFO refrigerants}

The most commonly used refrigerants in recent past were R11, R12, and R22 which because of their high ODP have been either phased out or under consideration for the same. After the revelation of the harmful effects of CFC and HCFC refrigerants on the ozone layer, search to find alternative working fluids gained more interest in the recent few years. The HFC134a was found to be a suitable candidate for replacing R12 and is being successfully used to date in small equipment like domestic refrigerators and water coolers and in mobile air-conditioning. HFC134a has very high GWP which is a matter of environmental concern [1]..HFO stands for hydro-fluoro-olefin (HFO-1234yf) is a low global warming potential (GWP) refrigerant for use in automotive air-conditioning systems. HFC-134a is a hydrofluoro-carbon refrigerant, while (HFO-1234yf) is a hydro-fluoroolefin refrigerant. Hydro-fluoro-olefin, or in short HFO, is a definition that is familiar to many of us. R1234yf, R1234ze are few examples of HFOs. They are used in a number of applications today, but have been barely studied just a decade agoHFO-1234yf was developed to meet the European directive 2006/40/EC in 2011 requiring use of HFO refrigerant in AC system with a GWP below 150. HFO-1234yf, which has a 100-year GWP lower than 1. These refrigerants are used as a "near drop-in replacement" for $\mathrm{R}-134 \mathrm{a}$, the current product used in automobile AC systems, which has a 100-year GWP of 1430. HFO-1234yf has the lowest cost among the currently proposed alternatives [2]. The initial cost of refrigeration and air conditioning system using R1234yf is much higher than that of R-134a and handled in repair shops in the same way as R-134a. Although it would require different, specialized equipment to perform the service due to the mild flammability of HFO-1234yf and another issue affecting the compatibility between HFO-1234yf and R-134a-based systems due to choice of lubricating oil. The current lubricating oil is showing signs of damage to plastic and aluminum, and issues with health, including mouth dryness, rashes, and sore throat, among other effects [3].

\section{Literature Review}

HFO-1234yf would be adopted as a replacement of R-134a automotive air-conditioning refrigerant.Mishra ${ }^{[2]}$ concluded that the first law efficiency in terms of coefficient of performance $\mathrm{COP}$ and second law efficiency in terms of exergetic efficiency of HFC-134a and HFO-1234ze is almost same having a difference of $5.6 \%$, which decreases with the increase in evaporator temperature, whereas it is $14.5-5 \%$ higher than HFO1234yf. Hence HFO-1234yf can be a good drop-in' replacement of HFC-134a at higher value of evaporator temperature and HFO$1234 \mathrm{ze}$ can be a good replacement after certain modification. From the irreversibility or exergy destruction viewpoint, worst component is condenser followed by compressor, throttle valve, evaporator and liquid vapour heat exchanger, the most efficient component. Total efficiency defect is more for HFO-1234yf followed by HFO-1234ze and HFC-134a, but the difference is small. Increase in ambient state temperature has a increasing (positive) effect on second law efficiency in terms of exergetic efficiency and exergy destruction ratio which was computed based on exergy of fuel or based on exergy of product (EDR). When exergy destruction ratio (EDR) reduced, then exergetic efficiency increases. Therefore HFO-1234yf gives lesser values of exergetic efficiency whereas HFO-1234ze gives approximately similar values.4. HFC-134a gives higher COP and exergetic efficiency than HFO-1234yf but lesser value than HFO1234ze. However reverse trend is seen when effectiveness of heat exchanger is increased from 0 to 1 . Hence, it can be concluded that even though the values of performance parameters for HFO1234yf are smaller than that of HFC-134a, but the difference is small, so it can a good alternative to HFC-134a because of its environmentally friendly properties. However HFO-1234ze can replace the conventional HFC-134a after having slight modification in the design as the performance parameters are almost similar [2].

\subsection{Energy_Exergy Analysis of Vapour Compression Refrigeration Systems}

The second law analysis (i.e. exergy Computation) is widely accepted as a useful tool for obtaining overall performances of any system for finding various exergy losses occurred in its components Exergy analysis also helps in taking account the important engineering decisions regarding design parameters of a system by finding maximum exergy destruction using entropy generation principle Many researchers have carried out exergy studies of different thermal energy conversion systems describing various approach for exergy analysis and its usefulness for improving existing designs by reducing exergy destruction in a more simple and effective manner [1-3] Padilla et al. ${ }^{[4]}$ computed the exergy performance of a domestic vapor compression refrigeration system (VCRS) by using zeotropic mixture (R413A) for direct replacement of R12 and found that the overall energy and exergy performances of this system working with R413A is far better than R12. Arora and Kaushik [5] presented a detailed exergy analysis of an actual vapour compression refrigeration (VCR) cycle and developed computational model for computing coefficient of performance (COP), exergy destruction, exergetic efficiency and efficiency defects for R502, R404A and R507A and found that the R507A is a better substitute to R502 than R404A. The efficiency defect in condenser is highest, and lowest in liquid vapour heat exchanger for R502, R404A and R507A refrigerants in the range of $-50^{\circ} \mathrm{C}$ to $0^{\circ} \mathrm{C}$ evaporator temperature and in the range and $40{ }^{\circ} \mathrm{C}$ to $55^{\circ} \mathrm{C}$ condenser temperature respectively. Anand $\mathrm{S}$ and Tyagi S. K. [6] presented a detailed experimental analysis of 2 ton of refrigeration capacity vapor compression refrigeration cycle using R22 as working fluid for different percentage of refrigerant charge using exergy analysis and evaluated thermal performances (i.e. coefficient of performance, exergy destruction, and exergetic efficiency) under variable quantity of refrigerant and found that the losses in the compressor are more pronounced, while the losses in the condenser are less pronounced as compared to other components. A computational model based on the exergy analysis is presented by Yumrutas et. al [7] for the investigation of the effects of the evaporating and condensing 
temperatures on the pressure losses, exergy losses, second law of efficiency, and the COP of a vapour compression cycle. Dincer ${ }^{[\underline{8}]}$ asserts that conventional energy analysis, based on the first law of thermodynamics, evaluates energy mainly on its quantity but analysis that are based on second law considers not only the quality of energy, but also quantity of energy. Kumar et al. [9] also carried out to carry out the exergetic analysis of a VCR system using R11 and R12 as refrigerants. Nikolaidis and Probert [10] used exergy method for computing thermodynamic performances of R22 in a two-stage compound compression cycle, with flash intercooling.

Getu and Bansal [11] had optimized the design and operating parameters of like condensing temperature, subcooling temperature, evaporating temperature, superheating temperature and temperature difference in cascade heat exchanger R744-R717 cascade refrigeration system. A regression analysis was also done to obtain optimum thermodynamic parameters of same system.

From the irreversibility or exergy destruction viewpoint, worst component is condenser followed by compressor, throttle valve, evaporator and liquid vapour heat exchanger, the most efficient component. Total efficiency defect is more for HFO-1234yf followed by HFO-1234ze and HFC-134a, but the difference is small. Increase in ambient state temperature has a increasing (positive) effect on second law efficiency in terms of exergetic efficiency and exergy destruction ratio which was computed based on exergy of fuel or based on exergy of product (EDR). When exergy destruction ratio (EDR) reduced, then the exergetic efficiency increases. Therefore HFO-1234yf gives lesser values of exergetic efficiency whereas HFO-1234ze gives approximately 4\% less values. HFC-134a gives higher COP and exergetic efficiency than HFO-1234yf but lesser value than HFO1234ze [12].

Based on the literature it was observed that Researchers have gone through detailed first law analysis in terms of coefficient of performance and second law analysis in term of exergetic efficiency of simple vapour compression refrigeration system with single evaporator. Authors also analyzed the effect of nanofluids on simple vapour compression cycle in the term of pool boiling, COP, Thermal conductivity etc Researchers did not go through the irreversibility analysis or second law analysis of multiple evaporators systems with multi-stage expansion in vapour compression refrigeration systems.

\section{Energy and exergy analysis}

For carrying out energetic and exergetic analysis, computational models of system-1 to system- 4 has been developed and impact of chosen refrigerants on these systems has been analyzed

Exergy at any state is given as

$\mathrm{X}=\left(\Phi-\Phi_{0}\right)-\mathrm{T}_{0}\left(\mathrm{~s}-\mathrm{s}_{0}\right)$

\subsection{Energy analysis}

First law of thermodynamic gives the idea of energy balance of system.

Mass flow analysis of systems

$$
\begin{aligned}
\dot{m}_{c 1} & =\dot{m}_{e 1}=\frac{\dot{Q}_{e 1}}{\left(\Phi_{1}-\Phi_{10}\right)} \\
\dot{m}_{e 2} & =\frac{\dot{Q}_{e 2}}{\left(\Phi_{3}-\Phi_{9}\right)} \\
\dot{m}_{f 1} & =\frac{\dot{m}_{c 1}\left(\Phi_{2}-\Phi_{3}\right)}{\left(\Phi_{3}-\Phi_{9}\right)} \\
\dot{m}_{c 2} & =\dot{m}_{c 1}+\dot{m}_{e 2}+\dot{m}_{f 1} \\
\dot{m}_{e 3} & =\frac{\dot{Q}_{e 3}}{\left(\Phi_{5}-\Phi_{8}\right)} \\
\dot{m}_{f 2} & =\frac{\dot{m}_{c 2}\left(\Phi_{4}-\Phi_{5}\right)}{\left(\Phi_{5}-\Phi_{8}\right)} \\
\dot{m}_{c 3} & =\dot{m}_{c 2}+\dot{m}_{e 3}+\dot{m}_{f 2}
\end{aligned}
$$

Energy consumption for systems

$$
\begin{aligned}
& P_{c 1}=\frac{\dot{m}_{c 1}\left(\Phi_{2}-\Phi_{1}\right)}{60} \\
& P_{c 2}=\frac{\dot{m}_{c 2}\left(\Phi_{4}-\Phi_{3}\right)}{60} \\
& P_{c 3}=\frac{\dot{m}_{c 3}\left(\Phi_{6}-\Phi_{5}\right)}{60}
\end{aligned}
$$

Energetic efficiency of system-1

$$
\mathrm{COP}=\frac{\dot{Q}_{e}}{P_{c} * 60}
$$

\subsection{Rate of exergy loss due to irreversibility $\left(T_{o} \dot{S}_{g e n}\right)$ in various components of system-1}

The concept of exergy was given by second law of thermodynamics, which always decreases due to thermodynamic irreversibility. Exergy is defined as the measure of usefulness, quality or potential of a stream to cause change and an effective measure of the potential of a substance to impact the environment [12].

\section{Compressors}

$$
\begin{aligned}
& \left(T_{o} \dot{S}_{g e n}\right)_{c 1}=\dot{W}_{c 1}+m_{c 1}\left(X_{2}-X_{1}\right) \\
& \left(T_{o} \dot{S}_{g e n}\right)_{c 2}=\dot{W}_{c 2} \cdot m_{c 2}\left(X_{4}-X_{3}\right) \\
& \left(T_{o} \dot{S}_{g e n}\right)_{c 3}=\dot{W}_{c 3}+m_{c 3}\left(X_{6}-X_{5}\right) \\
& \dot{\Psi}_{c}=\left(T_{o} \dot{S}_{g e n}\right)_{c 1}+\left(T_{o} \dot{S}_{g e n}\right)_{c 2}+\left(T_{o} \dot{S}_{g e n}\right)_{c 3}
\end{aligned}
$$


Evaporators

$$
\begin{aligned}
& \left(\mathrm{T}_{\mathrm{o}} \dot{\mathrm{S}}_{\mathrm{gen}}\right)_{\mathrm{e} 1}=\dot{\mathrm{m}}_{\mathrm{e} 1}\left(\mathrm{X}_{1}-\mathrm{X}_{10}\right)-\dot{\mathrm{Q}}_{\mathrm{e} 1}\left(1-\frac{\mathrm{T}_{0}}{\mathrm{~T}_{\mathrm{r} 1}}\right) \\
& \left(\mathrm{T}_{\mathrm{o}} \dot{\mathrm{S}}_{\mathrm{gen}}\right)_{\mathrm{e} 2}=\dot{\mathrm{m}}_{\mathrm{e} 2}\left(\mathrm{X}_{3}-\mathrm{X}_{9}\right)-\dot{\mathrm{Q}}_{\mathrm{e} 2}\left(1-\frac{\mathrm{T}_{0}}{\mathrm{~T}_{\mathrm{r} 2}}\right) \\
& \left(\mathrm{T}_{\mathrm{o}} \dot{\mathrm{S}}_{\mathrm{gen}}\right)_{\mathrm{e} 3}=\dot{\mathrm{m}}_{\mathrm{e} 3}\left(\mathrm{X}_{5}-\mathrm{X}_{8}\right)-\dot{\mathrm{Q}}_{\mathrm{e} 3}\left(1-\frac{\mathrm{T}_{0}}{\mathrm{~T}_{\mathrm{r} 3}}\right) \\
& \dot{\Psi}_{e}=\left(\mathrm{T}_{\mathrm{o}} \dot{\mathrm{S}}_{\mathrm{gen}}\right)_{\mathrm{e} 1}+\left(\mathrm{T}_{\mathrm{o}} \dot{\mathrm{S}}_{\mathrm{gen}}\right)_{\mathrm{e} 2}+\left(\mathrm{T}_{\mathrm{o}} \dot{\mathrm{S}}_{\text {gen }}\right)_{\mathrm{e} 3}
\end{aligned}
$$

$$
\begin{aligned}
& \text { Condenser } \\
& \begin{aligned}
\dot{\Psi}_{\text {cond }} & =\left(\mathrm{T}_{\mathrm{o}} \dot{\mathrm{S}}_{\text {gen }}\right)_{\text {cond }} \\
& =\dot{\mathrm{m}}_{\mathrm{c} 3}\left(\mathrm{X}_{6}-\mathrm{X}_{7}\right)-\dot{\mathrm{Q}}_{\mathrm{e}}\left(1-\frac{\mathrm{T}_{0}}{\mathrm{~T}_{\mathrm{r}}}\right)
\end{aligned}
\end{aligned}
$$

Throttle Valves

$$
\begin{gathered}
\left(\mathrm{T}_{\mathrm{o}} \dot{\mathrm{S}}_{\mathrm{gen}}\right)_{\mathrm{tv} 1}=\dot{\mathrm{m}}_{\mathrm{e} 1}\left(\mathrm{X}_{77}-\mathrm{X}_{10}\right) \\
\left(\mathrm{T}_{\mathrm{o}} \dot{\mathrm{S}}_{\mathrm{gen}}\right)_{\mathrm{tv} 2}=\left(\dot{\mathrm{m}}_{\mathrm{e} 2}+\dot{\mathrm{m}}_{\mathrm{f} 1}\right)\left(\mathrm{X}_{77}-\mathrm{X}_{9}\right) \\
\left(\mathrm{T}_{\mathrm{o}} \dot{\mathrm{S}}_{\mathrm{gen}}\right)_{\mathrm{tv} 3}=\left(\dot{\mathrm{m}}_{\mathrm{e} 3}+\dot{\mathrm{m}}_{\mathrm{f} 2}\right)\left(\mathrm{X}_{77}-\mathrm{X}_{8}\right) \\
\dot{\Psi}_{t v}=\left(\mathrm{T}_{\mathrm{o}} \dot{\mathrm{S}}_{\mathrm{gen}}\right)_{\mathrm{tv} 1}+\left(\mathrm{T}_{\mathrm{o}} \dot{\mathrm{S}}_{\mathrm{gen}}\right)_{\mathrm{tv} 2} \\
+\left(\mathrm{T}_{\mathrm{o}} \dot{\mathrm{S}}_{\mathrm{gen}}\right)_{\mathrm{tv} 3}
\end{gathered}
$$

\section{Liquid subcooler}

$$
\dot{\Psi}_{l s c}=\left(\mathrm{T}_{\mathrm{o}} \dot{\mathrm{S}}_{\mathrm{gen}}\right)_{\mathrm{sc}}=\dot{\mathrm{m}}_{\mathrm{c} 3}\left(\mathrm{X}_{7}-\mathrm{X}_{77}\right)
$$

Flash intercoolers

$$
\begin{aligned}
& \left(\mathrm{T}_{\mathrm{o}} \dot{\mathrm{S}}_{\mathrm{gen}}\right)_{\mathrm{f} 1}=\dot{\mathrm{m}}_{\mathrm{f} 1}\left(\mathrm{X}_{9}-\mathrm{X}_{3}\right)+\dot{\mathrm{m}}_{\mathrm{c} 1}\left(\mathrm{X}_{2}-\mathrm{X}_{3}\right) \\
& \left(\mathrm{T}_{\mathrm{o}} \dot{\mathrm{S}}_{\mathrm{gen}}\right)_{\mathrm{f} 2}=\dot{\mathrm{m}}_{\mathrm{f} 2}\left(\mathrm{X}_{8}-\mathrm{X}_{5}\right)+\dot{\mathrm{m}}_{\mathrm{c} 1}\left(\mathrm{X}_{4}-\mathrm{X}_{5}\right) \\
& \dot{\Psi}_{f}=\left(\mathrm{T}_{\mathrm{o}} \dot{\mathrm{S}}_{\mathrm{gen}}\right)_{\mathrm{f} 1}+\left(\mathrm{T}_{\mathrm{o}} \dot{\mathrm{S}}_{\mathrm{gen}}\right)_{\mathrm{f} 2}
\end{aligned}
$$

Total irreversibility destruction in system-1

$$
\begin{aligned}
& \sum \dot{\Psi}_{\mathrm{k}}=\dot{\Psi}_{\mathrm{e}}+\dot{\Psi}_{\mathrm{c}}+\dot{\Psi}_{\mathrm{cond}}+\dot{\Psi}_{\mathrm{tv}}+\dot{\Psi}_{\mathrm{lsc}}+\dot{\Psi}_{\mathrm{f}} \\
& \dot{m}_{c 1^{\prime}}=\dot{m}_{e 1^{\prime}}=\frac{\dot{Q}_{e 1^{\prime}}}{\left(\Phi_{1},-\Phi_{12^{\prime}}\right)} \\
& \dot{m}_{e 2^{\prime}}=\frac{\dot{Q}_{e 2^{\prime}}}{\left(\Phi_{3^{\prime}}-\Phi_{10^{\prime}}\right)}+\dot{m}_{c 1^{\prime}}\left(\frac{x_{10^{\prime}}}{1-x_{10^{\prime}}}\right) \\
& \dot{m}_{f 1^{\prime}}=\frac{\dot{m}_{c 1^{\prime}}\left(\Phi_{2^{\prime}}-\Phi_{3^{\prime}}\right)}{\left(\Phi_{3^{\prime}}-\Phi_{10^{\prime}}\right)}
\end{aligned}
$$

$$
\begin{aligned}
& \dot{m}_{c 2^{\prime}}=\dot{m}_{c 1^{\prime}}+\dot{m}_{e 2^{\prime}}+\dot{m}_{f 1^{\prime}} \\
& \dot{m}_{e 3^{\prime}}=\frac{\dot{Q}_{e 3^{\prime}}}{\left(\Phi_{5^{\prime}}-\Phi_{8^{\prime}}\right)}+\dot{m}_{c 2^{\prime}}\left(\frac{x_{8^{\prime}}}{1-x_{8^{\prime}}}\right) \\
& \dot{m}_{f 2^{\prime}}=\frac{\dot{m}_{c 2^{\prime}}\left(\Phi_{4^{\prime}}-\Phi_{5^{\prime}}\right)}{\left(\Phi_{5^{\prime}}-\Phi_{8^{\prime}}\right)}
\end{aligned}
$$

Power required for running the compressors

$$
\begin{aligned}
& P_{c 1^{\prime}}=\frac{\dot{m}_{c 1^{\prime}}\left(\Phi_{2^{\prime}}-\Phi_{1^{\prime}}\right)}{60} \\
& P_{c 2^{\prime}}=\frac{\dot{m}_{c 2^{\prime}}\left(\Phi_{4^{\prime}}-\Phi_{3^{\prime}}\right)}{60} \\
& P_{c 3^{\prime}}=\frac{\dot{m}_{c 3^{\prime}}\left(\Phi_{6^{\prime}}-\Phi_{5^{\prime}}\right)}{60}
\end{aligned}
$$

Energetic efficiency $=\frac{\dot{Q}_{e^{\prime}}}{P_{c}, * 60}$

3.3 Rate of exergy loss due to irreversibilties $\left(T_{o} \dot{S}_{g e n}\right)$ in various components of system-2

\section{Compressors}

$$
\begin{aligned}
& \left(\mathrm{T}_{\mathrm{o}} \dot{\mathrm{S}}_{\mathrm{gen}}\right)_{\mathrm{c} 1^{\prime}}=\dot{\mathrm{W}}_{\mathrm{c} 1^{\prime}}+\mathrm{m}_{\mathrm{c} 1^{\prime}}\left(\mathrm{X}_{2^{\prime}}-\mathrm{X}_{1^{\prime}}\right) \\
& \left(\mathrm{T}_{\mathrm{o}} \dot{\mathrm{S}}_{\mathrm{gen}}\right)_{\mathrm{c} 2^{\prime}}=\dot{\mathrm{W}}_{\mathrm{c} 2^{\prime}}{ }^{\prime}+\mathrm{m}_{\mathrm{c} 2^{\prime}}\left(\mathrm{X}_{4^{\prime}}-\mathrm{X}_{3^{\prime}}\right) \\
& \left(\mathrm{T}_{\mathrm{o}} \dot{\mathrm{S}}_{\mathrm{gen}}\right)_{\mathrm{c} 3^{\prime}}=\dot{\mathrm{W}}_{\mathrm{c} 3^{\prime}}+\mathrm{m}_{\mathrm{c} 3^{\prime}}\left(\mathrm{X}_{6^{\prime}}-\mathrm{X}_{5^{\prime}}\right) \\
& \dot{\Psi}_{\mathrm{c}^{\prime}}=\left(\mathrm{T}_{\mathrm{o}} \dot{\mathrm{S}}_{\mathrm{gen}}\right)_{\mathrm{c} 1^{\prime}}+\left(\mathrm{T}_{\mathrm{o}} \dot{\mathrm{S}}_{\mathrm{gen}}\right)_{\mathrm{c} 2^{\prime}} \\
& +\left(\mathrm{T}_{\mathrm{o}} \dot{\mathrm{S}}_{\mathrm{gen}}\right)_{\mathrm{c} 3^{\prime}}
\end{aligned}
$$

Evaporators

$$
\begin{aligned}
& \left(\mathrm{T}_{\mathrm{o}} \dot{\mathrm{S}}_{\mathrm{gen}}\right)_{\mathrm{e} 1^{\prime}}=\dot{\mathrm{m}}_{\mathrm{e} 1^{\prime}}\left(\mathrm{X}_{1^{\prime}}-\mathrm{X}_{12^{\prime}}-\dot{\mathrm{Q}}_{\mathrm{e} 1^{\prime}}\left(1-\frac{\mathrm{T}_{0}}{\mathrm{~T}_{\mathrm{r}^{\prime}}}\right)\right. \\
& \left(\mathrm{T}_{\mathrm{o}} \dot{\mathrm{S}}_{\mathrm{gen}}\right)_{\mathrm{e} 2^{\prime}}=\dot{\mathrm{m}}_{\mathrm{e} 2^{\prime}}\left(\mathrm{X}_{3^{\prime}}-\mathrm{X}_{10^{\prime}}\right)-\dot{\mathrm{Q}}_{\mathrm{e} 2^{\prime}}\left(1-\frac{\mathrm{T}_{0}}{\mathrm{~T}_{\mathrm{r} 2^{\prime}}}\right)
\end{aligned}
$$

$$
\begin{aligned}
& \left(\mathrm{T}_{\mathrm{o}} \dot{\mathrm{S}}_{\mathrm{gen}}\right)_{\mathrm{e}^{\prime}}=\dot{\mathrm{m}}_{\mathrm{e}^{\prime}}\left(\mathrm{X}_{5^{\prime}}-\mathrm{X}_{8^{\prime}}\right)-\dot{\mathrm{Q}}_{\mathrm{e} 3^{\prime}}\left(1-\frac{\mathrm{T}_{0}}{\mathrm{~T}_{\mathrm{r} 3^{\prime}}}\right) \\
& \dot{\Psi}_{\mathrm{e}^{\prime}}=\left(\mathrm{T}_{\mathrm{o}} \dot{\mathrm{S}}_{\mathrm{gen}}\right)_{\mathrm{e} 1^{\prime}}+\left(\mathrm{T}_{\mathrm{o}} \dot{\mathrm{S}}_{\mathrm{gen}}\right)_{\mathrm{e} 2^{\prime}}+\left(\mathrm{T}_{\mathrm{o}} \dot{\mathrm{S}}_{\mathrm{gen}}\right)_{\mathrm{e} 3^{\prime}}
\end{aligned}
$$

\section{Condenser}

$$
\begin{aligned}
& \dot{\Psi}_{\text {cond }}=\left(\mathrm{T}_{\mathrm{o}} \dot{\mathrm{S}}_{\text {gen }}\right)_{\text {cond }} \\
& =\dot{\mathrm{m}}_{\mathrm{c} 3^{\prime}}\left(\mathrm{X}_{6^{\prime}}-\mathrm{X}_{7^{\prime}}\right)-\dot{\mathrm{Q}}_{\mathrm{e}^{\prime}}\left(1-\frac{\mathrm{T}_{0}}{\mathrm{~T}_{\mathrm{r}^{\prime}}}\right)
\end{aligned}
$$


Throttle Valves

$\left(\mathrm{T}_{\mathrm{o}} \dot{\mathrm{S}}_{\mathrm{gen}}\right)_{\mathrm{tv} 1^{\prime}}=\dot{\mathrm{m}}_{\mathrm{e} 1^{\prime}}\left(\mathrm{X}_{11^{\prime}}-\mathrm{X}_{12^{\prime}}\right)$

$\left(\mathrm{T}_{\mathrm{o}} \dot{\mathrm{S}}_{\mathrm{gen}}\right)_{\mathrm{tv} 2^{\prime}}=\dot{\mathrm{m}}_{\mathrm{c} 2^{\prime}}\left(\mathrm{X}_{9^{\prime}}-\mathrm{X}_{10^{\prime}}\right)$

$\left(\mathrm{T}_{\mathrm{o}} \dot{\mathrm{S}}_{\mathrm{gen}}\right)_{\mathrm{tv} 3^{\prime}}=\dot{\mathrm{m}}_{\mathrm{c} 3^{\prime}}\left(\mathrm{X}_{77^{\prime}}-\mathrm{X}_{8^{\prime}}\right)$

$\dot{\Psi}_{\mathrm{tv}}=\left(\mathrm{T}_{\mathrm{o}} \dot{\mathrm{S}}_{\mathrm{gen}}\right)_{\mathrm{tv} 1^{\prime}}+\left(\mathrm{T}_{\mathrm{o}} \dot{\mathrm{S}}_{\mathrm{gen}}\right)_{\mathrm{tv} 2^{\prime}}+\left(\mathrm{T}_{\mathrm{o}} \dot{\mathrm{S}}_{\mathrm{gen}}\right)_{\mathrm{tv} 3^{\prime}}(53)$

\section{Liquid subcooler}

$\dot{\Psi}_{l \mathrm{sc}}=\left(\mathrm{T}_{\mathrm{o}} \dot{\mathrm{S}}_{\mathrm{gen}}\right)_{\mathrm{lsc}}=\dot{\mathrm{m}}_{\mathrm{c} 3^{\prime}}\left(\mathrm{X}_{7^{\prime}}-\mathrm{X}_{77^{\prime}}\right)$

Flash intercoolers

$\left(\mathrm{T}_{\mathrm{o}} \dot{\mathrm{S}}_{\mathrm{gen}}\right)_{\mathrm{f} 1^{\prime}}=\dot{\mathrm{m}}_{\mathrm{f} 1^{\prime}}\left(\mathrm{X}_{10^{\prime}}-\mathrm{X}_{3^{\prime}}\right)+\dot{\mathrm{m}}_{\mathrm{c} 1^{\prime}}\left(\mathrm{X}_{2^{\prime}}-\mathrm{X}_{3^{\prime}}\right)$

$\left(\mathrm{T}_{\mathrm{o}} \dot{\mathrm{S}}_{\mathrm{gen}}\right)_{\mathrm{f} 2^{\prime}}=\dot{\mathrm{m}}_{\mathrm{f} 2^{\prime}}\left(\mathrm{X}_{8^{\prime}}-\mathrm{X}_{5^{\prime}}\right)+\dot{\mathrm{m}}_{\mathrm{c} 2^{\prime}}\left(\mathrm{X}_{4^{\prime}}-\mathrm{X}_{5^{\prime}}\right)$

$\dot{\Psi}_{\mathrm{f}^{\prime}}=\left(\mathrm{T}_{\mathrm{o}} \dot{\mathrm{S}}_{\mathrm{gen}}\right)_{\mathrm{f} 1^{\prime}}+\left(\mathrm{T}_{\mathrm{o}} \dot{\mathrm{S}}_{\mathrm{gen}}\right)_{\mathrm{f} 2^{\prime}}$

Total irreversibility destruction in system-1

$\sum \dot{\Psi}_{\mathrm{k}^{\prime}}=\dot{\Psi}_{\mathrm{e}^{\prime}}+\dot{\Psi}_{\mathrm{c}^{\prime}}+\dot{\Psi}_{\mathrm{cond}^{\prime}}+\dot{\Psi}_{\mathrm{tv}^{\prime}}+\dot{\Psi}_{\mathrm{lsc}^{\prime}}+\dot{\Psi}_{\mathrm{f}^{\prime}}$

Exergetic efficiency

$=\frac{\text { Exergy of cooling load of evaporators }}{\text { Compressors work }}=\frac{\dot{E} P}{\dot{W}}$

Exergetic efficiency of system

$=\frac{\left(\dot{\mathrm{Q}}_{\mathrm{e} 1}+\dot{\mathrm{Q}}_{\mathrm{e} 2}+\dot{\mathrm{Q}}_{\mathrm{e} 3}\right)-\mathrm{T}_{\mathrm{o}}\left(\frac{\dot{\mathrm{Q}}_{\mathrm{e} 1}}{\mathrm{~T}_{\mathrm{r} 1}}+\frac{\dot{\mathrm{Q}}_{\mathrm{e} 2}}{\mathrm{~T}_{\mathrm{r} 2}}+\frac{\dot{\mathrm{Q}}_{\mathrm{e} 3}}{\mathrm{~T}_{\mathrm{r} 3}}\right)}{P_{C} * 60}$

Rational efficiency of system -2

$=\frac{\left(\dot{\mathrm{Q}}_{\mathrm{e} 1^{\prime}}+\dot{\mathrm{Q}}_{\mathrm{e} 2^{\prime}}+\dot{\mathrm{Q}}_{\mathrm{e} 3^{\prime}}\right)-\mathrm{T}_{\mathrm{o}}\left(\frac{\dot{\mathrm{Q}}_{\mathrm{e}^{\prime}}}{\mathrm{T}_{\mathrm{r} 1^{\prime}}}+\frac{\dot{\mathrm{Q}}_{\mathrm{e}^{\prime}}}{\mathrm{T}_{\mathrm{r} 2^{\prime}}}+\frac{\dot{\mathrm{Q}}_{\mathrm{e} 3^{\prime}}}{\mathrm{T}_{\mathrm{r} 3^{\prime}}}\right)}{\mathrm{P}_{\mathrm{c}^{\prime}} * 60}$

3.4 Rate of exergy loss due to irreversibility $\left(T_{o} \dot{S}_{g e n}\right)$ in various components of system-3

Compressors

$$
\begin{aligned}
& \left(T_{o} \dot{S}_{g e n}\right)_{c 1}=\dot{W}_{c 1}+m_{c 1}\left(X_{2}-X_{1}\right) \\
& \left(T_{o} \dot{S}_{g e n}\right)_{c 2}=\dot{W}_{c 2} \dot{+}+m_{c 2}\left(X_{4}-X_{3}\right) \\
& \left(T_{o} \dot{S}_{g e n}\right)_{c 3}=\dot{W}_{c 3} \cdot m_{c 3}\left(X_{6}-X_{5}\right) \\
& \dot{\Psi}_{c}=\left(T_{o} \dot{S}_{g e n}\right)_{c 1}+\left(T_{o} \dot{S}_{g e n}\right)_{c 2}+\left(T_{o} \dot{S}_{g e n}\right)_{c 3}
\end{aligned}
$$

Evaporators

$$
\begin{aligned}
& \left(\mathrm{T}_{\mathrm{o}} \dot{\mathrm{S}}_{\mathrm{gen}}\right)_{\mathrm{e} 1}=\dot{\mathrm{m}}_{\mathrm{e} 1}\left(\mathrm{X}_{1}-\mathrm{X}_{10}\right)-\dot{\mathrm{Q}}_{\mathrm{e} 1}\left(1-\frac{\mathrm{T}_{0}}{\mathrm{~T}_{\mathrm{r} 1}}\right) \\
& \left(\mathrm{T}_{\mathrm{o}} \dot{\mathrm{S}}_{\mathrm{gen}}\right)_{\mathrm{e} 2}=\dot{\mathrm{m}}_{\mathrm{e} 2}\left(\mathrm{X}_{3}-\mathrm{X}_{9}\right)-\dot{\mathrm{Q}}_{\mathrm{e} 2}\left(1-\frac{\mathrm{T}_{0}}{\mathrm{~T}_{\mathrm{r} 2}}\right) \\
& \left(\mathrm{T}_{\mathrm{o}} \dot{\mathrm{S}}_{\mathrm{gen}}\right)_{\mathrm{e} 3}=\dot{\mathrm{m}}_{\mathrm{e} 3}\left(\mathrm{X}_{5}-\mathrm{X}_{8}\right)-\dot{\mathrm{Q}}_{\mathrm{e} 3}\left(1-\frac{\mathrm{T}_{0}}{\mathrm{~T}_{\mathrm{r} 3}}\right) \\
& \dot{\Psi}_{e}=\left(\mathrm{T}_{\mathrm{o}} \dot{\mathrm{S}}_{\mathrm{gen}}\right)_{\mathrm{e} 1}+\left(\mathrm{T}_{\mathrm{o}} \dot{\mathrm{S}}_{\mathrm{gen}}\right)_{\mathrm{e} 2}+\left(\mathrm{T}_{\mathrm{o}} \dot{\mathrm{S}}_{\mathrm{gen}}\right)_{\mathrm{e} 3}
\end{aligned}
$$

Condenser

$$
\begin{aligned}
\dot{\Psi}_{\text {cond }} & =\left(\mathrm{T}_{\mathrm{o}} \dot{\mathrm{S}}_{\text {gen }}\right)_{\text {cond }} \\
& =\dot{\mathrm{m}}_{\mathrm{c} 3}\left(\mathrm{X}_{6}-\mathrm{X}_{7}\right)-\dot{\mathrm{Q}}_{\mathrm{e}}\left(1-\frac{\mathrm{T}_{0}}{\mathrm{~T}_{\mathrm{r}}}\right)
\end{aligned}
$$

Throttle Valves

$$
\begin{gathered}
\left(\mathrm{T}_{\mathrm{o}} \dot{\mathrm{S}}_{\mathrm{gen}}\right)_{\mathrm{tv} 1}=\dot{\mathrm{m}}_{\mathrm{e} 1}\left(\mathrm{X}_{77}-\mathrm{X}_{10}\right) \\
\left(\mathrm{T}_{\mathrm{o}} \dot{\mathrm{S}}_{\mathrm{gen}}\right)_{\mathrm{tv} 2}=\left(\dot{\mathrm{m}}_{\mathrm{e} 2}+\dot{\mathrm{m}}_{\mathrm{f} 1}\right)\left(\mathrm{X}_{77}-\mathrm{X}_{9}\right) \\
\left(\mathrm{T}_{\mathrm{o}} \dot{\mathrm{S}}_{\mathrm{gen}}\right)_{\mathrm{tv} 3}=\left(\dot{\mathrm{m}}_{\mathrm{e} 3}+\dot{\mathrm{m}}_{\mathrm{f} 2}\right)\left(\mathrm{X}_{77}-\mathrm{X}_{8}\right) \\
\dot{\Psi}_{t v}=\left(\mathrm{T}_{\mathrm{o}} \dot{\mathrm{S}}_{\mathrm{gen}}\right)_{\mathrm{tv} 1}+\left(\mathrm{T}_{\mathrm{o}} \dot{\mathrm{S}}_{\mathrm{gen}}\right)_{\mathrm{tv} 2} \\
+\left(\mathrm{T}_{\mathrm{o}} \dot{\mathrm{S}}_{\text {gen }}\right)_{\mathrm{tv} 3}
\end{gathered}
$$

\section{Liquid subcooler}

$$
\begin{aligned}
& \dot{\Psi}_{l s c}=\left(\mathrm{T}_{\mathrm{o}} \dot{\mathrm{S}}_{\mathrm{gen}}\right)_{\mathrm{sc}}=\dot{\mathrm{m}}_{\mathrm{c} 3}\left(\mathrm{X}_{7}-\mathrm{X}_{77}\right) \\
& \dot{\Psi}_{f}=\left(\mathrm{T}_{\mathrm{o}} \dot{\mathrm{S}}_{\mathrm{gen}}\right)_{\mathrm{f} 1}+\left(\mathrm{T}_{\mathrm{o}} \dot{\mathrm{S}}_{\mathrm{gen}}\right)_{\mathrm{f} 2}
\end{aligned}
$$

Total irreversibility destruction in system-3

$\sum \dot{\Psi}_{\mathrm{k}}=\dot{\Psi}_{\mathrm{e}}+\dot{\Psi}_{\mathrm{c}}+\dot{\Psi}_{\mathrm{cond}}+\dot{\Psi}_{\mathrm{tv}}+\dot{\Psi}_{\mathrm{lsc}}+\dot{\Psi}_{\mathrm{f}}$

$\dot{m}_{c 1}=\dot{m}_{e 1}=\frac{\dot{Q}_{e 1}}{\left(\Phi_{1},-\Phi_{12}\right)}$

$\dot{m}_{e 2^{\prime}}=\frac{\dot{Q}_{e 2^{\prime}}}{\left(\Phi_{3},-\Phi_{10}\right)}+\dot{m}_{c 1^{\prime}}\left(\frac{x_{10^{\prime}}}{1-x_{10^{\prime}}}\right)$

$\dot{m}_{f 1}=\frac{\dot{m}_{c 1}\left(\Phi_{2},-\Phi_{3}\right)}{\left(\Phi_{3},-\Phi_{10}\right)}$

$\dot{m}_{c 2^{\prime}}=\dot{m}_{c 1^{\prime}}+\dot{m}_{e 2},+\dot{m}_{f 1}$

$\dot{m}_{e 3^{\prime}}=\frac{\dot{Q}_{e 3^{\prime}}}{\left(\Phi_{5^{\prime}}-\Phi_{8}\right)}+\dot{m}_{c 2^{\prime}}\left(\frac{x_{8^{\prime}}}{1-x_{8^{\prime}}}\right)$ 
$\dot{m}_{f 2}=\frac{\dot{m}_{c 2^{\prime}}\left(\Phi_{4},-\Phi_{5^{\prime}}\right)}{\left(\Phi_{5},-\Phi_{8}\right)}$

Power required for running the compressors

$$
\begin{aligned}
P_{c 1^{\prime}} & =\frac{\dot{m}_{c 1^{\prime}}\left(\Phi_{2^{\prime}}-\Phi_{1^{\prime}}\right)}{60} \\
P_{c 2^{\prime}} & =\frac{\dot{m}_{c 2^{\prime}}\left(\Phi_{4^{\prime}}-\Phi_{3^{\prime}}\right)}{60} \\
P_{c 3^{\prime}} & =\frac{\dot{m}_{c 3^{\prime}}\left(\Phi_{6^{\prime}}-\Phi_{5^{\prime}}\right)}{60}
\end{aligned}
$$

Energetic efficiency $=\frac{\dot{Q}_{e^{\prime}}}{P_{c} * 60}$

3.5 Rate of exergy loss due to irreversibilties $\left(T_{o} \dot{S}_{g e n}\right)$ in various components of system-4

\section{Compressors}

$$
\begin{gathered}
\left(\mathrm{T}_{\mathrm{o}} \dot{\mathrm{S}}_{\mathrm{gen}}\right)_{\mathrm{c} 1^{\prime}}=\dot{\mathrm{W}}_{\mathrm{c} 1^{\prime}}+\mathrm{m}_{\mathrm{c} 1^{\prime}}\left(\mathrm{X}_{2^{\prime}}-\mathrm{X}_{1^{\prime}}\right) \\
\left(\mathrm{T}_{\mathrm{o}} \dot{\mathrm{S}}_{\mathrm{gen}}\right)_{\mathrm{c} 2^{\prime}}=\dot{\mathrm{W}}_{\mathrm{c} 2^{\prime}} \dot{\mathrm{m}_{\mathrm{c} 2^{\prime}}}\left(\mathrm{X}_{4^{\prime}}-\mathrm{X}_{3^{\prime}}\right) \\
\left(\mathrm{T}_{\mathrm{o}} \dot{\mathrm{S}}_{\mathrm{gen}}\right)_{\mathrm{c} 3^{\prime}}=\dot{\mathrm{W}}_{\mathrm{c} 3^{\prime}} \dot{\mathrm{m}_{\mathrm{c} 3^{\prime}}}\left(\mathrm{X}_{6^{\prime}}-\mathrm{X}_{5^{\prime}}\right) \\
\dot{\Psi}_{\mathrm{c}^{\prime}}=\left(\mathrm{T}_{\mathrm{o}} \dot{\mathrm{S}}_{\mathrm{gen}}\right)_{\mathrm{c} 1^{\prime}}+\left(\mathrm{T}_{\mathrm{o}} \dot{\mathrm{S}}_{\mathrm{gen}}\right)_{\mathrm{c} 2^{\prime}} \\
+\left(\mathrm{T}_{\mathrm{o}} \dot{\mathrm{S}}_{\mathrm{gen}}\right)_{\mathrm{c} 3^{\prime}}
\end{gathered}
$$

\section{Evaporators}

$$
\begin{aligned}
& \left(\mathrm{T}_{\mathrm{o}} \dot{\mathrm{S}}_{\mathrm{gen}}\right)_{\mathrm{e} 1^{\prime}}=\dot{\mathrm{m}}_{\mathrm{e}^{\prime}}\left(\mathrm{X}_{1^{\prime}}-\mathrm{X}_{12^{\prime}}-\dot{\mathrm{Q}}_{\mathrm{e}^{\prime}}\left(1-\frac{\mathrm{T}_{0}}{\mathrm{~T}_{\mathrm{r} 1^{\prime}}}\right)\right. \\
& \left(\mathrm{T}_{\mathrm{o}} \dot{\mathrm{S}}_{\mathrm{gen}}\right)_{\mathrm{e} 2^{\prime}}=\dot{\mathrm{m}}_{\mathrm{e} 2^{\prime}}\left(\mathrm{X}_{3^{\prime}}-\mathrm{X}_{10^{\prime}}\right)-\dot{\mathrm{Q}}_{\mathrm{e} 2^{\prime}}\left(1-\frac{\mathrm{T}_{0}}{\mathrm{~T}_{\mathrm{r} 2^{\prime}}}\right) \\
& \left(\mathrm{T}_{\mathrm{o}} \dot{\mathrm{S}}_{\mathrm{gen}}\right)_{\mathrm{e} 3^{\prime}}=\dot{\mathrm{m}}_{\mathrm{e} 3^{\prime}}\left(\mathrm{X}_{5^{\prime}}-\mathrm{X}_{8^{\prime}}\right)-\dot{\mathrm{Q}}_{\mathrm{e} 3^{\prime}}\left(1-\frac{\mathrm{T}_{0}}{\mathrm{~T}_{\mathrm{r} 3^{\prime}}}\right) \\
& \dot{\Psi}_{\mathrm{e}^{\prime}}=\left(\mathrm{T}_{\mathrm{o}} \dot{\mathrm{S}}_{\mathrm{gen}}\right)_{\mathrm{e} 1^{\prime}}+\left(\mathrm{T}_{\mathrm{o}} \dot{\mathrm{S}}_{\mathrm{gen}}\right)_{\mathrm{e} 2^{\prime}}+\left(\mathrm{T}_{\mathrm{o}} \dot{\mathrm{S}}_{\mathrm{gen}}\right)_{\mathrm{e} 3^{\prime}}
\end{aligned}
$$

\section{Condenser}

$$
\begin{aligned}
& \dot{\Psi}_{\text {cond }}=\left(\mathrm{T}_{\mathrm{o}} \dot{\mathrm{S}}_{\text {gen }}\right)_{\text {cond }} \\
& =\dot{\mathrm{m}}_{\mathrm{c} 3^{\prime}}\left(\mathrm{X}_{6^{\prime}}-\mathrm{X}_{7^{\prime}}\right)-\dot{\mathrm{Q}}_{\mathrm{e}^{\prime}}\left(1-\frac{\mathrm{T}_{0}}{\mathrm{~T}_{\mathrm{r}^{\prime}}}\right)
\end{aligned}
$$

Throttle Valves

$$
\left(\mathrm{T}_{\mathrm{o}} \dot{\mathrm{S}}_{\mathrm{gen}}\right)_{\mathrm{tv} 1^{\prime}}=\dot{\mathrm{m}}_{\mathrm{e} 1^{\prime}}\left(\mathrm{X}_{11^{\prime}}-\mathrm{X}_{12^{\prime}}\right)
$$

$$
\begin{aligned}
& \left(\mathrm{T}_{\mathrm{o}} \dot{\mathrm{S}}_{\mathrm{gen}}\right)_{\mathrm{tv} 2^{\prime}}=\dot{\mathrm{m}}_{\mathrm{c} 2^{\prime}}\left(\mathrm{X}_{9^{\prime}}-\mathrm{X}_{10^{\prime}}\right) \\
& \left(\mathrm{T}_{\mathrm{o}} \dot{\mathrm{S}}_{\mathrm{gen}}\right)_{\mathrm{tv} 3^{\prime}}=\dot{\mathrm{m}}_{\mathrm{c}^{\prime}}\left(\mathrm{X}_{77^{\prime}}-\mathrm{X}_{8^{\prime}}\right) \\
& \dot{\Psi}_{\mathrm{tv}{ }^{\prime}}=\left(\mathrm{T}_{\mathrm{o}} \dot{\mathrm{S}}_{\mathrm{gen}}\right)_{\mathrm{tv} 1^{\prime}}+\left(\mathrm{T}_{\mathrm{o}} \dot{\mathrm{S}}_{\mathrm{gen}}\right)_{\mathrm{tv} 2^{\prime}}+\left(\mathrm{T}_{\mathrm{o}} \dot{\mathrm{S}}_{\mathrm{gen}}\right)_{\mathrm{tv} 3^{\prime}}
\end{aligned}
$$

Liquid subcooler

$\dot{\Psi}_{l \mathrm{sc}}=\left(\mathrm{T}_{\mathrm{o}} \dot{\mathrm{S}}_{\mathrm{gen}}\right)_{\mathrm{lsc}^{\prime}}=\dot{\mathrm{m}}_{\mathrm{c} 3^{\prime}}\left(\mathrm{X}_{7^{\prime}}-\mathrm{X}_{77^{\prime}}\right)$

Total irreversibility destruction in system-1

$\sum \dot{\Psi}_{\mathrm{k}^{\prime}}=\dot{\Psi}_{\mathrm{e}^{\prime}}+\dot{\Psi}_{\mathrm{c}^{\prime}}+\dot{\Psi}_{\mathrm{cond}^{\prime}}+\dot{\Psi}_{\mathrm{tv}^{\prime}}+\dot{\Psi}_{\mathrm{lsc}^{\prime}}+\dot{\Psi}_{\mathrm{f}^{\prime}}$

Exergetic efficiency for system-4 can be expressed as Exergetic efficiency

$$
=\frac{\text { Exergy of cooling load of evaporators }}{\text { Compressors work }}=\frac{\dot{E P}}{\dot{W}}
$$

Exergetic efficiency of system -4 is

$$
=\frac{\left(\dot{\mathrm{Q}}_{\mathrm{e} 1}+\dot{\mathrm{Q}}_{\mathrm{e} 2}+\dot{\mathrm{Q}}_{\mathrm{e} 3}\right)-\mathrm{T}_{\mathrm{o}}\left(\frac{\dot{\mathrm{Q}}_{\mathrm{e} 1}}{\mathrm{~T}_{\mathrm{r} 1}}+\frac{\dot{\mathrm{Q}}_{\mathrm{e} 2}}{\mathrm{~T}_{\mathrm{r} 2}}+\frac{\dot{\mathrm{Q}}_{\mathrm{e} 3}}{\mathrm{~T}_{\mathrm{r} 3}}\right)}{P_{c} * 60}
$$

\section{Results and Discussion}

Following systems have been considered for present investigations.

System-1: Vapour compression refrigeration system using multiple evaporators at different temperatures with compound compression, individual expansion valves and flash inter coolers System-2: Vapour compression refrigeration system using multiple evaporators at different temperatures with compound compression, multiple expansion valves and flash inter coolers System-3: Vapour compression refrigeration system using multiple evaporators at different temperatures with individual compressors, multiple expansion valves

System-4: Vapour compression refrigeration system using multiple evaporators at different temperatures with individual compressors, individual expansion valves.

Comparison were made of for above four systems with following input conditions using ecofriendly refrigerants for following input conditions

- Load on the low, intermediate and high temperature evaporators are 30TR, $20 \mathrm{TR}$ and $10 \mathrm{TR}$ respectively.

- Dead state temperature $\left(\mathrm{T}_{0}\right): 25^{\circ} \mathrm{C}$

- Difference between evaporator and space temperature $\left(\mathrm{T}_{\mathrm{r}^{-}}\right.$ $\left.\mathrm{T}_{\mathrm{e}}\right): 5{ }^{\circ} \mathrm{C}$.

- Adiabatic efficiency of compressor: $80 \%$.

- Dead state enthalpy $\left(\Phi_{0}\right)$ and entropy $\left(\mathrm{s}_{0}\right)$ of the refrigerants have been calculated corresponding to the dead state temperature $\left(\mathrm{T}_{0}\right)$ of $25^{\circ} \mathrm{C}$. 
- Variation in kinetic and potential energy is negligible.

- Expansion process is adiabatic

- Temperature of low, intermediate and high temperature evaporators are $-5^{\circ} \mathrm{C}, 0^{\circ} \mathrm{C}$ and $5^{\circ} \mathrm{C}$ respectively.

- Condenser temperature : $40^{\circ} \mathrm{C}$

- Degree of sub cooling : $10^{\circ} \mathrm{C}$

It is clear from Table-1, that vapour compression refrigeration system using R134a with multiple evaporators at different temperatures with individual compressors, multiple expansion valves (system-3) gives better thermodynamic performances with low electrical power required to run whole system) i.e. all compressors. It is also observed that exergy destruction in condenser is highest. However the exergy destruction in all compressors is almost similar. Similarly exergy destruction in condensers in all almost similar. The exergy destruction in evaporators in System-2: Vapour compression refrigeration system using multiple evaporators at different temperatures with compound compression, multiple expansion valves and flash inter coolers( system-2) is highest as compared to other systems. How ever exergy destruction in evaporators system-3 has less exergy destruction than system- 2 but higher than system- 1 and system-4. It is also observed that exergy destruction in flash chamber is lowest. exergy destruction in expansion valves of system-4 is higher than system-1, system-2 and system3.

Table 1(a): Thermodynamic performances of various vapour compression refrigeration systems using HFC-134a refrigerant

\begin{tabular}{|c|c|c|c|c|}
\hline Performance parameters & System-1 & System-2 & System-3 & System-4 \\
\hline First Law efficiency (COP_Actual) & 4.297 & 4.428 & 4.593 & 4.291 \\
\hline Exergy Destruction Ratio (EDR) & 1.961 & 2 & 1.894 & 1.965 \\
\hline Exergetic Efficiency using First Method & 0.3377 & 0.3481 & 0.361 & 0.3373 \\
\hline Exergy of Fuel $(\mathrm{kW})$ & 48.87 & 47.42 & 45.72 & 48.94 \\
\hline Exergy of Product(kW) & 16.51 & 16.51 & 16.51 & 16.51 \\
\hline Exergetic Efficiency using second Method & 0.3329 & 0.3311 & 0.3108 & 0.3322 \\
\hline Sum of total Exergy Destruction in three Compressors (\%) & 23.82 & 23.82 & 23.15 & 23.13 \\
\hline Exergy Destruction in Condensers & 24.28 & 24.5 & 24.89 & 24.23 \\
\hline Sum of total Exergy Destruction in three Evaporators (\%) & 7.411 & 14.45 & 14.07 & 7.218 \\
\hline Sum of total Exergy Destruction in three Expansion Valves (\%) & 8.91 & 4.857 & 4.322 & 9.823 \\
\hline Exergy Destruction in Subcooler (\%) & 1.749 & 1.945 & 1.931 & 1.871 \\
\hline Exergy Destruction in Flash chambers $(\%)$ & 0.0513 & 0.042 & Flash chambers not used & Not used Flash chambers \\
\hline Second law efficiency & 0.481 & 0.5073 & 0.5141 & 0.4803 \\
\hline
\end{tabular}

\subsection{Effect of different Load conditions}

Comparison were made of for above four systems with input conditions using ecofriendly refrigerants for following input conditions

1. Load on the low, intermediate and high temperature evaporators are 10TR, $20 \mathrm{TR}$ and $30 \mathrm{TR}$ respectively.

2. Dead state temperature $\left(\mathrm{T}_{0}\right): 25^{\circ} \mathrm{C}$ (i.e. $298 \mathrm{~K}$ )

3. Difference between evaporator and space temperature $\left(\mathrm{T}_{\mathrm{r}}-\mathrm{T}_{\mathrm{e}}\right): 5^{\circ} \mathrm{C}$.
4. Adiabatic efficiency of compressor: $100 \%$

5. Dead state enthalpy $\left(\Phi_{0}\right)$ and entropy $\left(\mathrm{s}_{0}\right)$ of the refrigerants have been calculated corresponding to the dead state temperature $\left(\mathrm{T}_{0}\right)$ of $25^{\circ} \mathrm{C}$ (i.e. 298K)

6. Variation in kinetic and potential energy is negligible.

7. Expansion process is adiabatic

8. Temperature of low, intermediate and high temperature evaporators are $-5^{\circ} \mathrm{C}, 0^{\circ} \mathrm{C}$ and $5{ }^{\circ} \mathrm{C}$ respectively.

9. Condenser temperature : $40^{\circ} \mathrm{C}$

10. Degree of sub cooling : $10^{\circ} \mathrm{C}$

Table 1(b): Thermodynamic performances of various vapour compression refrigeration systems using HFC-134a refrigerant

\begin{tabular}{|c|c|c|c|c|}
\hline Performance parameters & System-1 & System-2 & System-3 & System-4 \\
\hline First Law efficiency (COP_Actual) & 4.807 & 4.87 & 4.983 & 4.79 \\
\hline Exergetic Efficiency using First Method & 0.3161 & 0.3202 & 0.3276 & 0.3149 \\
\hline Exergy of Product $(\mathrm{kW})$ & 12.6 & 12.6 & 12.6 & 12.6 \\
\hline Exergetic Efficiency using second Method & 0.3161 & 0.2953 & 0.3007 & 0.3149 \\
\hline Total Exergy Destruction in Compressors (\%) & 23.53 & 23.57 & 23.21 & 23.2 \\
\hline Exergy Destruction in Expansion Valves(\%) & 6.615 & 4.653 & 4.31 & 7.25 \\
\hline Exergy Destruction in Subcooler(\%) & 1.902 & 2.105 & 2.1 & 2.063 \\
\hline Exergy Destruction in Flash chambers (\%) & 0.08 & 0.02 & Not used Flash chambers & Not used Flash chambers \\
\hline
\end{tabular}

Comparison were made of for above four systems withinput conditions using ecofriendly refrigerants for following input conditions
- Load on the low, intermediate and high temperature evaporators are 20TR, $30 \mathrm{TR}$ and $10 \mathrm{TR}$ respectively.

- Dead state temperature $\left(\mathrm{T}_{0}\right): 25^{\circ} \mathrm{C}$ 
- Difference between evaporator and space temperature $\left(\mathrm{T}_{\mathrm{r}^{-}}\right.$ $\left.\mathrm{T}_{\mathrm{e}}\right): 5^{\circ} \mathrm{C}$.

- Adiabatic efficiency of compressor: $75 \%$.

- Dead state enthalpy $\left(\Phi_{0}\right)$ and entropy $\left(\mathrm{s}_{0}\right)$ of the refrigerants have been calculated corresponding to the dead state temperature $\left(\mathrm{T}_{0}\right)$ of $25^{\circ} \mathrm{C}$.
- Variation in kinetic and potential energy is negligible.

- Expansion process is adiabatic

- Temperature of low, intermediate and high temperature evaporators are $-5{ }^{\circ} \mathrm{C}, 0{ }^{\circ} \mathrm{C}$ and $5{ }^{\circ} \mathrm{C}$ respectively.

- Condenser temperature : $40^{\circ} \mathrm{C}$

- Degree of sub cooling : $10^{\circ} \mathrm{C}$

Table 1(c): Thermodynamic performances of various vapour compression refrigeration systems using HFC-134a refrigerant

\begin{tabular}{|c|c|c|c|c|}
\hline Performance parameters & System-1 & System-2 & System-3 & System-4 \\
\hline First Law efficiency (COP_Actual) & 4.419 & 4.532 & 4.699 & 4.41 \\
\hline Exergy Destruction Ratio (EDR) & 2.004 & 2.047 & 1.947 & 2.01 \\
\hline Exergetic Efficiency using First Method & 0.3329 & 0.3414 & 0.354 & 0.3322 \\
\hline Exergy of Fuel $(\mathrm{kW})$ & 43.02 & 40.85 & 40.54 & 42.63 \\
\hline Exergy of Product $(\mathrm{kW})$ & 12.6 & 12.6 & 12.6 & 12.6 \\
\hline Exergy Destruction in Compressors (\%) & 23.75 & 23.5 & 23.17 & 23.15 \\
\hline Exergy Destruction in Condensers (\%) & 24.84 & 24.73 & 25.32 & 24.75 \\
\hline Exergy Destruction Evaporators (\%) & 8.044 & 7.91 & 14.18 & 7.9 \\
\hline Exergy Destruction in Expansion Valves (\%) & 8.247 & 9.07 & 4.28 & 9.076 \\
\hline Exergy Destruction in Subcooler $(\%)$ & 1.784 & 1.92 & 1.97 & 1.917 \\
\hline Exergy Destruction in Flash chambers (\%) & 0.05 & 0.051 & Not used Flash chambers & Not used Flash chambers \\
\hline Exergetic efficiency second methods efficiency & 0.3329 & 0.3311 & 0.3108 & 0.3322 \\
\hline
\end{tabular}

\subsection{Effect of HFO-1234ze ecofriendly refrigerant}

Case-1: In this investigation following assumptions are made by using HFO-1234ze refrigerant shown in table-2. Similarly thermodynamic performances of various systems using R1234ze are shown in Table-2(b) to Table-2(d) respectively.

Table-2(a): Thermodynamic performances of various vapour compression refrigeration systems using HFO-1234ze refrigerant

\begin{tabular}{|c|c|c|c|c|}
\hline Input Data & System-1 & System-2 & System-3 & System-4 \\
\hline Refrigerant & HFO-1234ze & HFO-1234ze & HFO-1234ze & HFO-1234ze \\
\hline Q_Eva1 $(\mathrm{kW})$ & 70 & 105 & 70 & 70 \\
\hline Q_Eva2 $(\mathrm{kW})$ & 105 & 70 & 105 & 105 \\
\hline Q_Eva3 $(\mathrm{kW})$ & 35 & 35 & 35 & 35 \\
\hline Temperature of Evaporator-1 $\left({ }^{\circ} \mathrm{C}\right)$ & -5 & -10 & -10 & -10 \\
\hline Temperature of Evaporator-2 $\left({ }^{\circ} \mathrm{C}\right)$ & 0 & 5 & 5 & 5 \\
\hline Temperature of Evaporator-3 $\left({ }^{\circ} \mathrm{C}\right)$ & 5 & 10 & 10 & 10 \\
\hline Temperature of condenser $\left({ }^{\circ} \mathrm{C}\right)$ & 40 & 40 & 40 & 40 \\
\hline Subcooling Temperature of condenser $\left({ }^{\circ} \mathrm{C}\right)$ & 30 & 30 & 30 & 30 \\
\hline Isentropic efficiency of compressors & 0.75 & 0.75 & 0.75 & 0.75 \\
\hline
\end{tabular}

vapour compression refrigeration system using multiple evaporators at different temperatures with individual compressors, multiple expansion valves (system-3) gives better thermodynamic performances with low electrical power required to run whole system) i.e. all compressors. It is also observed that exergy destruction in condenser is highest. However the exergy destruction in all compressors is almost similar. Similarly exergy destruction in condensers in all almost similar. The exergy destruction in evaporators in System-2: Vapour compression refrigeration system using multiple evaporators at different temperatures with compound compression, multiple expansion valves and flash inter coolers( system-2) is highest as compared to other systems. How ever exergy destruction in evaporators system-3 has less exergy destruction than system-2 but higher than system-1 and system-4. It is also observed that exergy destruction in flash chamber is lowest. exergy destruction in expansion valves of system-4 is higher than system-1, system-2 and system 3 .

Table 2(b): Thermodynamic performances of various vapour compression refrigeration systems using HFO-1234ze refrigerant

\begin{tabular}{|c|c|c|c|c|}
\hline Performance parameters & System-1 & System-2 & System-3 & System-4 \\
\hline First Law efficiency (COP_Actual) & 4.881 & 5.141 & 5.18 & 4.926 \\
\hline Exergy Destruction Ratio (EDR) & 2.415 & 2.338 & 2.313 & 2.383 \\
\hline Exergetic Efficiency using First Method & 0.2929 & 0.3085 & 0.3108 & 0.2956 \\
\hline Exergy of Fuel (kW) & 43.02 & 40.85 & 40.54 & 42.63 \\
\hline Exergy of Product(kW) & 12.6 & 12.6 & 12.6 & 12.6 \\
\hline Exergetic Efficiency using second Method & 0.2929 & 0.2789 & 0.2956 & 0.2956 \\
\hline Second law efficiency (\%) & 64.96 & 68.42 & 0.6556 & 0.6556 \\
\hline
\end{tabular}


Table 2(c): Exergy_Destruction of various components in vapour compression refrigeration systems using HFO-1234ze refrigerant

\begin{tabular}{|c|c|c|c|c|}
\hline Performance parameters & System-1 & System-2 & System-3 & System-4 \\
\hline Total Exergy Destruction in compressors (\%) & 24.31 & 24.21 & 23.69 & 23.69 \\
\hline Total Exergy Destruction in condensers (\%) & 27.68 & 28.9 & 29.12 & 27.93 \\
\hline Total Exergy Destruction in evaporators (\%) & 7.756 & 11.12 & 11.2 & 7.827 \\
\hline Total Exergy Destruction in expansion Valves (\%) & 8.405 & 5.192 & 5.2 & 8.432 \\
\hline Total Exergy Destruction in sub coolers & 2.565 & 2.698 & 2.68 & 2.568 \\
\hline Rational Efficiency (\%) & 29.29 & 27.89 & 29.56 & 29.56 \\
\hline
\end{tabular}

Table (2d): Work done by each compressor in vapour compression refrigeration systems using HFO-1234ze refrigerant

\begin{tabular}{|c|c|c|c|c|}
\hline Performance parameters & System-1 & System-2 & System-3 & System-4 \\
\hline Work done by compressor $-1(\mathrm{~kW})$ & 2.498 & 1.862 & 8.025 & 10.77 \\
\hline Work done by compressor $-2(\mathrm{~kW})$ & 6.767 & 5.517 & 13.4 & 15.7 \\
\hline Work done by compressor 3 $(\mathrm{kW})$ & 33.76 & 33.47 & 19.1 & 16.16 \\
\hline Exergy of Fuel $(\mathrm{kW})$ & 43.02 & 40.85 & 40.54 & 42.63 \\
\hline Exergy of Product $(\mathrm{kW})$ & 12.6 & 12.6 & 12.6 & 12.6 \\
\hline Exergetic Efficiency using second Method $(\%)$ & 29.29 & 30.85 & 28.11 & 29.56 \\
\hline
\end{tabular}

Case-2: In this investigation following assumptions are made by using HFO refrigerant shown in Table-3(a)

Table 3: Thermodynamic performances of various vapour compression refrigeration systems using HFO-1234yf refrigerant

\begin{tabular}{|c|c|c|c|c|}
\hline Input Data & System-1 & System-2 & System-3 & System-4 \\
\hline Refrigerant & R1234yf & R1234yf & R1234yf & R1234yf \\
\hline Q_Eva1 $(\mathrm{kW})$ & 70 & 105 & 70 & 70 \\
\hline Q_Eva2 $(\mathrm{kW})$ & 105 & 70 & 105 & 105 \\
\hline Q_Eva3 $(\mathrm{kW})$ & 35 & 35 & 35 & 35 \\
\hline Temperature of Evaporator-1 $\left({ }^{\circ} \mathrm{C}\right)$ & -5 & -10 & -10 & -10 \\
\hline Temperature of Evaporator-2 $\left({ }^{\circ} \mathrm{C}\right)$ & 0 & 5 & 5 & 5 \\
\hline Temperature of Evaporator-3 $\left({ }^{\circ} \mathrm{C}\right)$ & 5 & 10 & 10 & 10 \\
\hline Temperature of condenser $\left({ }^{\circ} \mathrm{C}\right)$ & 40 & 40 & 40 & 40 \\
\hline Subcooling Temperature of condenser $\left({ }^{\circ} \mathrm{C}\right)$ & 30 & 30 & 30 & 30 \\
\hline Isentropic efficiency of compressors & 0.75 & 0.75 & 0.75 & 0.75 \\
\hline
\end{tabular}

vapour compression refrigeration system using multiple evaporators at different temperatures with individual compressors, multiple expansion valves (system-3) gives better thermodynamic performances with low electrical power required to run whole system) i.e. all compressors. It is also observed that exergy destruction in condenser is highest. However the exergy destruction in all compressors is almost similar. Similarly exergy destruction in condensers in all almost similar. The exergy destruction in evaporators in System-2: Vapour compression refrigeration system using multiple evaporators at different temperatures with compound compression, multiple expansion valves and flash inter coolers( system-2) is highest as compared to other systems. How ever exergy destruction in evaporators system-3 has less exergy destruction than system-2 but higher than system-1 and system-4. It is also observed that exergy destruction in flash chamber is lowest. exergy destruction in expansion valves of system-4 is higher than system-1, system-2 and system 3 .

Case-3: In this investigation following assumptions are made by using HFC-1234yf refrigerant:

Table 3(a): Thermodynamic performances of various vapour compression refrigeration systems using HFO-134a refrigerant

\begin{tabular}{|c|c|c|c|c|}
\hline Input Data & System-1 & System-2 & System-3 & System-4 \\
\hline Refrigerant & R134a & R134a & R134a & R134a \\
\hline Q_Eva1 $(\mathrm{kW})$ & 70 & 105 & 70 & 70 \\
\hline Q_Eva2 $(\mathrm{kW})$ & 105 & 70 & 105 & 105 \\
\hline Q_Eva3 $(\mathrm{kW})$ & 35 & 35 & 35 & 35 \\
\hline Temperature of Evaporator-1 $\left({ }^{\circ} \mathrm{C}\right)$ & -5 & -10 & -10 & -10 \\
\hline Temperature of Evaporator-2 $\left({ }^{\circ} \mathrm{C}\right)$ & 0 & 5 & 5 & 5 \\
\hline Temperature of Evaporator-3 $\left({ }^{\circ} \mathrm{C}\right)$ & 5 & 10 & 10 & 10 \\
\hline Temperature of condenser $\left({ }^{\circ} \mathrm{C}\right)$ & 40 & 40 & 40 & 40 \\
\hline Subcooling Temperature of condenser $\left({ }^{\circ} \mathrm{C}\right)$ & 30 & 30 & 30 & 30 \\
\hline Isentropic efficiency of compressors & 0.75 & 0.75 & 0.75 & 0.75 \\
\hline
\end{tabular}


The exergy destruction in condenser is higher than compressor. For comparing all four systems, the exergy destruction in system3 is high and lower in system-4 respectively. The exergy destruction in running all compressors of system-1 is highest while for system- 4 is lowest. However the exergy destruction in all compressors is almost similar. Similarly exergy destruction in condenser in all systems is almost similar. The exergy destruction in evaporators in System-2: Vapour compression refrigeration system using multiple evaporators at different temperatures with compound compression, multiple expansion valves and flash inter coolers (system-2) is highest as compared to other systems. However exergy destruction in evaporators system-3 has less exergy destruction than system-2 but higher than system-1 and system-4. It is also observed that exergy destruction in flash chamber is lowest. exergy destruction in expansion valves of system-4 is higher than system-1, system- 2 and system 3 .

Table-3(a) shows the exergy destruction in various components of vapour compression refrigeration systems using R-134a refrigerant and it is observed that thermal performances (first and second law performances) vapour compression refrigeration system using multiple evaporators at different temperatures with compound compression, multiple expansion valves (system-3) is highest and Vapour compression refrigeration system using multiple evaporators at different temperatures with compound compression, individual expansion valves and flash inter coolers (system-1) is lowest.

Table 3(b): Thermodynamic performances of various vapour compression refrigeration systems using HFC-1234yf refrigerant)

\begin{tabular}{|c|c|c|c|c|}
\hline Performance parameters & System-1 & System-2 & System-3 & System-4 \\
\hline First Law efficiency (COP_Actual) & 4.972 & 5.188 & 5.227 & 5.017 \\
\hline Exergy Destruction Ratio (EDR) & 2.352 & 2.299 & 2.274 & 2.322 \\
\hline Exergetic Efficiency using First Method & 0.2983 & 0.3113 & 0.3136 & 0.301 \\
\hline Exergy of Fuel (kW) & 42.24 & 40.48 & 40.17 & 41.86 \\
\hline Exergy of Product(kW) & 12.6 & 12.6 & 12.6 & 12.6 \\
\hline Exergetic Efficiency using second Method & 0.2983 & 2845 & 0.2868 & 0.301 \\
\hline
\end{tabular}

Table-3(b) shows the exergy destruction in various components of vapour compression refrigeration systems using R-134a refrigerant and it is observed that Vapour compression refrigeration system using multiple evaporators at different temperatures with compound compression, multiple expansion valves and flash inter coolers (system-2) gives highest exergy destruction as compared to other systems.

Table 3(c): Exergy_Destruction of various components in vapour compression refrigeration systems using HFC-1234yf refrigerant

\begin{tabular}{|c|c|c|c|c|}
\hline Performance parameters & System-1 & System-2 & System-3 & System-4 \\
\hline Total Exergy Destruction in compressors (\%) & 23.98 & 23.89 & 23.22 & 23.19 \\
\hline Total Exergy Destruction in condensers (\%) & 26.81 & 27.78 & 28.25 & 27.35 \\
\hline Total Exergy Destruction in evaporators (\%) & 9.512 & 12.61 & 12.69 & 9.598 \\
\hline Total Exergy Destruction in expansion Valves (\%) & 7.632 & 4.968 & 4.942 & 7.61 \\
\hline Total Exergy Destruction in sub coolers & 2.166 & 2.244 & 2.23 & 2.149 \\
\hline Rational Efficiency (\%) & 29.83 & 28.45 & 28.68 & 30.1 \\
\hline
\end{tabular}

Table-3(c) shows the exergy destruction in various components of vapour compression refrigeration systems using R-245fa refrigerant and it is observed that exergy of fuel (Electrical power required to run all compressors) of vapour compression refrigeration system using multiple evaporators at different temperatures with compound compression, multiple expansion valves (system-3) is lowest and Vapour compression refrigeration system using multiple evaporators at different temperatures with compound compression, individual expansion valves and flash inter coolers (system-1) is highest. It is also observed that compressor-3 gives high work done by compressor while compressor give low work done.

Table 3(d): Work done by each compressor in vapour compression refrigeration systems using HFC-1234yf refrigerant

\begin{tabular}{|c|c|c|c|c|}
\hline Performance parameters & System-1 & System-2 & System-3 & System-4 \\
\hline Work done by compressor $-1(\mathrm{~kW})$ & 2.361 & 1.855 & 8.192 & 10.46 \\
\hline Work done by compressor $-2(\mathrm{~kW})$ & 6.504 & 5.489 & 13.52 & 15.4 \\
\hline Work done by compressor $3(\mathrm{~kW})$ & 33.37 & 33.13 & 18.46 & 16.01 \\
\hline Exergy of Fuel $(\mathrm{kW})$ & 42.24 & 40.48 & 40.17 & 41.86 \\
\hline Exergy of Product $(\mathrm{kW})$ & 12.6 & 12.6 & 12.6 & 12.6 \\
\hline Exergetic Efficiency using second Method $(\%)$ & 29.83 & 28.45 & 28.68 & 30.1 \\
\hline
\end{tabular}


Table 4: Input conditions for evaluating thermodynamic performances of various vapour compression refrigeration systems using $R$-236fa refrigerant

\begin{tabular}{|c|c|c|c|c|}
\hline Input Data & System-1 & System-2 & System-3 & System-4 \\
\hline Refrigerant & R-236fa & R-236fa & R-236fa & R-236fa \\
\hline Q_Eva1 $(\mathrm{kW})$ & 70 & 105 & 70 & 70 \\
\hline Q_Eva2 $(\mathrm{kW})$ & 105 & 70 & 105 & 105 \\
\hline Q_Eva3 $(\mathrm{kW})$ & 35 & 35 & 35 & 35 \\
\hline Temperature of Evaporator-1 $\left({ }^{\circ} \mathrm{C}\right)$ & -5 & -10 & -10 & -10 \\
\hline Temperature of Evaporator-2 $\left({ }^{\circ} \mathrm{C}\right)$ & 0 & 5 & 5 & 5 \\
\hline Temperature of Evaporator-3 $\left({ }^{\circ} \mathrm{C}\right)$ & 5 & 10 & 10 & 10 \\
\hline Temperature of condenser $\left({ }^{\circ} \mathrm{C}\right)$ & 40 & 40 & 40 & 40 \\
\hline Subcooling Temperature of condenser $\left({ }^{\circ} \mathrm{C}\right)$ & 30 & 30 & 30 & 30 \\
\hline Isentropic efficiency of compressors & 0.75 & 0.75 & 0.75 & 0.75 \\
\hline
\end{tabular}

Table-4(a) shows the exergy destruction in various components of vapour compression refrigeration systems using R-236fa refrigerant and it is observed that thermal performances (first and second law performances) vapour compression refrigeration system using multiple evaporators at different temperatures with compound compression, multiple expansion valves (system-3) is highest and Vapour compression refrigeration system using multiple evaporators at different temperatures with compound compression, individual expansion valves and flash inter coolers (system-1) is lowest. Table-4(b) shows the exergy destruction in various components of vapour compression refrigeration systems using R-236fa refrigerant and it is observed that Vapour compression refrigeration system using multiple evaporators at different temperatures with compound compression, multiple expansion valves and flash inter coolers (system-2) gives highest exergy dstruction as compared to other systems. The exergy destruction in condenser is higher than compressor. For comparing all four systems, the exergy destruction in system-3 is high and lower in system-4 respectively. The exergy destruction in running all compressors of system-1 is highest while for system-4 is lowest. However the exergy destruction in all compressors is almost similar. Similarly exergy destruction in condenser in all systems is almost similar. The exergy destruction in evaporators in System-2: Vapour compression refrigeration system using multiple evaporators at different temperatures with compound compression, multiple expansion valves and flash inter coolers (system-2) is highest as compared to other systems. However exergy destruction in evaporator's system-3 has less exergy destruction than system-2but higher than system-1 and system-4. It is also observed that exergy destruction in flash chamber is lowest. exergy destruction in expansion valves of system-4 is higher than system-1, system-2 and system 3 . Table4(c) shows the exergy destruction in various components of vapour compression refrigeration systems using R-236fa refrigerant and it is observed that exergy of fuel (Electrical power required to run all compressors) of vapour compression refrigeration system using multiple evaporators at different temperatures with compound compression, multiple expansion valves (system-3) is lowest and Vapour compression refrigeration system using multiple evaporators at different temperatures with compound compression, individual expansion valves and flash inter coolers (system-1) is highest. It is also observed that compressor-3 gives high work done by compressor while compressor give low work done.

Table4 (a): Thermodynamic performances of various vapour compression refrigeration systems using $R$-236fa refrigerant)

\begin{tabular}{|c|c|c|c|c|}
\hline Performance parameters & System-1 & System-2 & System-3 & System-4 \\
\hline First Law efficiency (COP_Actual) & 4.973 & 5.226 & 5.264 & 5.182 \\
\hline Exergy Destruction Ratio (EDR) & 2.352 & 2.287 & 2.264 & 2.217 \\
\hline Exergetic Efficiency using First Method & 0.2984 & 0.3136 & 0.3158 & 0.3109 \\
\hline Exergy of Fuel (kW) & 42.23 & 40.18 & 39.89 & 40.53 \\
\hline Exergy of Product(kW) & 12.6 & 12.6 & 12.6 & 12.6 \\
\hline Exergetic Efficiency using second Method & 0.2984 & 0.2829 & 0.285 & 0.3109 \\
\hline
\end{tabular}

Table 4(b): Exergy_Destruction of various components in vapour compression refrigeration systems using R-236fa refrigerant

\begin{tabular}{|c|c|c|c|c|}
\hline Performance parameters & System-1 & System-2 & System-3 & System-4 \\
\hline Total Exergy Destruction in compressors (\%) & 24.4 & 24.3 & 23.8 & 23.65 \\
\hline Total Exergy Destruction in condensers (\%) & 26.12 & 27.23 & 27.4 & 27.6 \\
\hline Total Exergy Destruction in evaporators (\%) & 9.545 & 13.1 & 13.19 & 9.944 \\
\hline Total Exergy Destruction in expansion Valves (\%) & 8.057 & 4.951 & 4.974 & 6.198 \\
\hline Total Exergy Destruction in sub coolers & 2.035 & 2.121 & 2.13 & 1.513 \\
\hline Rational Efficiency (\%) & 0.2984 & 28.29 & 28.5 & 0.3109 \\
\hline
\end{tabular}


Table 4(c): Work done by each compressor in vapour compression refrigeration systems using HFC-236fa refrigerant

\begin{tabular}{|c|c|c|c|c|}
\hline Performance parameters & System-1 & System-2 & System-3 & System-4 \\
\hline Work done by compressor $-1(\mathrm{~kW})$ & 2.46 & 1.854 & 8.094 & 10.09 \\
\hline Work done by compressor $-2(\mathrm{~kW})$ & 6.648 & 5.476 & 13.28 & 14.9 \\
\hline Work done by compressor 3 $(\mathrm{kW})$ & 33.12 & 32.85 & 17.69 & 15.53 \\
\hline Exergy of Fuel $(\mathrm{kW})$ & 42.23 & 40.18 & 39.89 & 40.53 \\
\hline Exergy of Product $(\mathrm{kW})$ & 12.6 & 12.6 & 12.6 & 12.6 \\
\hline Exergetic Efficiency using second Method $(\%)$ & 29.84 & 28.29 & 28.5 & 0.3109 \\
\hline
\end{tabular}

Case-4: In this investigation following assumptions are made by using $R$-245fa refrigerant:

Table 5: Input conditions for evaluating thermodynamic performances of various vapour compression refrigeration systems using $R-245$ fa refrigerant

\begin{tabular}{|c|c|c|c|c|}
\hline Input Data & System-1 & System-2 & System-3 & System-4 \\
\hline Refrigerant & R-245fa & R-245fa & R-245fa & R-245fa \\
\hline Q_Eva1 $(\mathrm{kW})$ & 70 & 105 & 70 & 70 \\
\hline Q_Eva2 $(\mathrm{kW})$ & 105 & 70 & 105 & 105 \\
\hline Q_Eva3 $(\mathrm{kW})$ & 35 & 35 & 35 & 35 \\
\hline Temperature of Evaporator-1 $\left({ }^{\circ} \mathrm{C}\right)$ & -5 & -10 & -10 & -10 \\
\hline Temperature of Evaporator-2 $\left({ }^{\circ} \mathrm{C}\right)$ & 0 & 5 & 5 & 5 \\
\hline Temperature of Evaporator-3 $\left({ }^{\circ} \mathrm{C}\right)$ & 5 & 10 & 10 & 10 \\
\hline Temperature of condenser $\left({ }^{\circ} \mathrm{C}\right)$ & 40 & 40 & 40 & 40 \\
\hline Subcooling Temperature of condenser $\left({ }^{\circ} \mathrm{C}\right)$ & 30 & 30 & 30 & 30 \\
\hline Isentropic efficiency of compressors & 0.75 & 0.75 & 0.75 & 0.75 \\
\hline
\end{tabular}

Table5 (a): Thermodynamic performances of various vapour compression refrigeration systems using $R$-245fa refrigerant)

\begin{tabular}{|c|c|c|c|c|}
\hline Performance parameters & System-1 & System-2 & System-3 & System-4 \\
\hline First Law efficiency (COP_Actual) & 5.137 & 5.337 & 5.376 & 5.182 \\
\hline Exergy Destruction Ratio (EDR) & 2.245 & 2.201 & 2.178 & 2.217 \\
\hline Exergetic Efficiency using First Method & 0.3082 & 0.3202 & 0.3226 & 0.3109 \\
\hline Exergy of Fuel (kW) & 40.88 & 39.35 & 39.06 & 40.53 \\
\hline Exergy of Product(kW) & 12.6 & 12.6 & 12.6 & 12.6 \\
\hline Exergetic Efficiency using second Method & 0.3082 & 0.2951 & 0.2975 & 0.3109 \\
\hline
\end{tabular}

Table-5(a) shows the exergy destruction in various components of vapour compression refrigeration systems using R-245fa refrigerant and it is observed that thermal performances (first and second law performances) vapour compression refrigeration system using multiple evaporators at different temperatures with compound compression, multiple expansion valves (system-3) is highest and Vapour compression refrigeration system using multiple evaporators at different temperatures with compound compression, individual expansion valves and flash inter coolers (system-1) is lowest. Table-5(b) shows the exergy destruction in various components of vapour compression refrigeration systems using R-245fa refrigerant and it is observed that Vapour compression refrigeration system using multiple evaporators at different temperatures with compound compression, multiple expansion valves and flash inter coolers (system-2) gives highest exergy dstruction as compared to other systems.The exergy destruction in condenser is higher than compressor. For comparing all four systems, the exergy destruction in system-3 is high and lower in system-4 respectively. The exergy destruction in running all compressors of system-1 is highest while for system-4 is lowest. However the exergy destruction in all compressors isalmost similar. Similarly exergy destruction in condenser in all systems is almost similar. The exergy destruction in evaporators in System-2: Vapour compression refrigeration system using multiple evaporators at different temperatures with compound compression, multiple expansion valves and flash inter coolers (system-2) is highest as compared to other systems. However exergy destruction in evaporators system-3 has less exergy destruction than system- 2 but higher than system- 1 and system-4. It is also observed that exergy destruction in flash chamber is lowest. exergy destruction in expansion valves of system- 4 is higher than system-1, system- 2 and system3.Table5 (c) shows the exergy destruction in various components of vapour compression refrigeration systems using R-245fa refrigerant and it is observed that exergy of fuel (Electrical power required to run all compressors) of vapour compression refrigeration system using multiple evaporators at different temperatures with compound compression, multiple expansion valves (system-3) is lowest and Vapour compression refrigeration system using multiple evaporators at different temperatures with compound compression, individual expansion valves and flash inter coolers (system-1) is highest. It is also observed that compressor-3 gives high work done by compressor while compressor 1 gives low compressor work. 
Table 5(b): Exergy_Destruction of various components in vapour compression refrigeration systems using $R$-245fa refrigerant

\begin{tabular}{|c|c|c|c|c|}
\hline Performance parameters & System-1 & System-2 & System-3 & System-4 \\
\hline Total Exergy Destruction in compressors (\%) & 24.3 & 24.22 & 23.66 & 23.65 \\
\hline Total Exergy Destruction in condensers (\%) & 27.29 & 28.18 & 28.45 & 27.6 \\
\hline Total Exergy Destruction in evaporators (\%) & 9.857 & 12.74 & 12.83 & 9.944 \\
\hline Total Exergy Destruction in expansion Valves (\%) & 6.188 & 3.744 & 3.743 & 6.198 \\
\hline Total Exergy Destruction in sub coolers & 1.516 & 1.565 & 1.563 & 1.513 \\
\hline Rational Efficiency (\%) & 30.82 & 0.2951 & 0.2975 & 31.09 \\
\hline
\end{tabular}

Table 5(c): Work done by each compressor in vapour compression refrigeration systems using HFC-245fa refrigerant

\begin{tabular}{|c|c|c|c|c|}
\hline Performance parameters & System-1 & System-2 & System-3 & System-4 \\
\hline Work done by compressor -1 $(\mathrm{kW})$ & 2.28 & 1.828 & 8.094 & 10.09 \\
\hline Work done by compressor -2 $(\mathrm{kW})$ & 6.268 & 5.387 & 13.28 & 14.9 \\
\hline Work done by compressor 3 $(\mathrm{kW})$ & 32.33 & 32.14 & 17.69 & 15.53 \\
\hline Exergy of Fuel $(\mathrm{kW})$ & 40.88 & 39.35 & 39.06 & 40.53 \\
\hline Exergy of Product $(\mathrm{kW})$ & 12.6 & 12.6 & 12.6 & 12.6 \\
\hline Exergetic Efficiency using second Method $(\%)$ & 30.82 & 29.51 & 29.75 & 31.09 \\
\hline
\end{tabular}

\section{Conclusions and Recommendations}

Following conclusions were drawn from present investigations

- Thermodynamic performance using R245fa gives better first and second law performances than R236fa and R134a. The performance of HFO refrigerants is slightly less than HFC134a however it has similar refrigerating properties and can easily replace HFC-134a in all multiple evaporators \& multiple compressorsand multiple expansion valves vapour compression refrigeration systems.

- The exergy destruction in condenser is highestin all four systems.

- The exergy destruction in compressors is higher than evaporators in all systems.

- The exergy of fuel (Electrical power required to run all compressors) of vapour compression refrigeration system using multiple evaporators at different temperatures with compound compression, multiple expansion valves (system3 ) is lowest and Vapour compression refrigeration system using multiple evaporators at different temperatures with compound compression, individual expansion valves and flash inter coolers (system-1) is highest.

- Highest work done by compressor-3 while compressor-1 delivered low compressor work.

\section{References}

[1] Johnson. E. Global warming from HFC. Environ. Impact Asses.1998; 18:485-492.

[2] Kapil Chopra, V.Sahni, R.S Mishra. Thermodynamic analyses of multiple evaporators vapour compression refrigeration systems with R410A, R290, R1234YF, R502, R404A and R152A.International Journal of Airconditioning and Refrigeration 21(1) (2014) 1-14.
[3] QiyuChen,R.C Prasad. Simulation of a vapour compression refrigeration cycles HFC134A and CFC12.Int Comm.Heat Mass Transfer.1999; 26:513-521.

[4] M. Padilla, R. Revellin, J. Bonjour. Exergy analysis of R413A as replacement of R12 in a domestic refrigeration system. Int J Energy Conversion and Management.2010; 51:2195-2201.

[5] AkhileshArora, S.C. Kaushik. Theoretical analysis of a vapour compression refrigeration system with R502, R404A and R507A. Int J Refrigeration.2008; 31:998-1005.

[6] S.Anand ,S.KTyagi-Exergy analysis and experimental study of a vapour compression refrigeration cycle.Int J Therm Anal Calorim.2012; 110:961971.

[7] RecepYumrutas, Mehmet Kunduz, Mehmet Kanoglu-Exergy analysis of vapor compression refrigeration systems. Exergy, An International Journal.2002; 2:266-272.

[8] Dincer.I. Refrigeration Systems and Applications. Wiley, UK.2003:26.

[9] S.Kumar, M.Prevost, R.Bugarel- Exergy analysis of a vapour compression refrigeration system. Heat Recovery Systems \& CHP.1989;9:151-157.

[10] C. Nikolaidis, D. Probert-Exergy method analysis of a two-stage vapourcompression refrigeration-plants performance. Int $\mathrm{J}$ Applied Thermal Engineering. 1998; 60:241-256.

[11] H.M Getu, P.K Bansal. Thermodynamic analysis of an R744-R717 cascade refrigeration system. Int J Refrigeration.2008; 31:45-54.

[12] Kapil Chopra, V.Sahni, R.S Mishra -Thermodynamic analysis of a multievaporators vapour compression refrigeration system using ecofriendly refrigerants (R1234yf, R1234ze and R134a). International Conference on Smart Technologies for Mechanical Engineers.2013.Paper No-42.

[13] Kapil Chopra, V.Sahni , R.S Mishra - Energy and exergy analysis of multiple evaporators and compressors with individual or multiple expansion valves vapour compression refrigeration system using ecofriendly refrigerants. International Conference on Smart Technologies for Mechanical Engineers.2013.Paper No-121.

[14] Klein, S.A., AlvaradoF. Engineering Equation Solver, Version 7.441. F Chart Software, Middleton, WI.2005.

Cite this article as: R.S. Mishra, Evaluation of thermodynamic energy-exergy performances in modified vapour compression refrigeration systems, International Journal of Research in Engineering and Innovation Vol-3, Issue-6 (2019), 364-376. . http://doi.org/10.36037/IJREI.2019.3603 